\title{
Nutcracker syndrome in a 13-year-old girl with haematuria - case report
}

\author{
Joanna Lechańska-Helman', Joanna Balcerak', Kamila Malewska-Kaczmarek1, \\ Paulina Kwarta', Agnieszka Szadkowska², Iwona Stelmach'1 \\ 'Department of Paediatrics and Allergy, Korczak Paediatric Centre, Copernicus Memorial Hospital in Lodz, \\ Medical University of Lodz, Poland \\ 2Department of Pediatrics, Oncology, Hematology, and Diabetology, Medical University of Lodz, Poland
}

\section{ABSTRACT}

Nutcracker syndrome (NCS) is an unusual cause of haematuria and is defined as compression of the left renal vein between the aorta and superior mesenteric artery, resulting in elevation of left renal vain pressure and collateral vein development. Symptoms of NCS include the following: haematuria, abdominal and left flank pain, pelvic congestion syndrome, and orthostatic proteinuria, although, clinically, gross haematuria may be the only disorder. We report a 13-year-old girl who experienced haematuria for two years with chronic back and abdominal pain. Each urinalysis revealed haematuria (20\% dysmorphic). In the presented case Doppler ultrasonography revealed left renal vein dilatation up to the angle between the superior mesenteric artery and the aorta with a diameter of $7 \mathrm{~mm}$ in the hilar region. NCS can be easily missed by routine tests in children.

\section{KEY WORDS:}

abdominal pain, paediatrics, haematuria, nutcracker syndrome.

\section{INTRODUCTION}

Intermittent haematuria in children is a common cause of hospitalisation, with a long list of aetiology factors including: urinary infections, hypercalciuria, nephrolithiasis, and glomerulonephritis.

Left renal vein (LRV) entrapment syndrome, commonly called nutcracker syndrome (NCS), is an unusual cause of haematuria. NCS is defined as compression of LRV between the aorta and superior mesenteric artery (SMA), which can lead to an increase in LRV pressure and development of collateral circulation. Routine diagnostic tools are often insufficient to make a diagnosis, so children with clinical features of NCS undergo repeated diagnostic procedures, which leads to delayed diagnosis. A helpful and non-invasive method that can be used initially for detecting rare causes of haematuria is Doppler ultrasonography (DUS), with 78\% sensitivity and 100\% specificity for NCS [1].

We report a 13-year-old girl who experienced haematuria with chronic back and abdominal pain.

\section{CASE REPORT}

A 13-year-old girl with intermittent haematuria that was observed for two years, and chronic back and abdominal pain for six months, was admitted to hospital. From the medical interview, it was known that symptoms exac-

\section{ADDRESS FOR CORRESPONDENCE:}

Joanna Lechańska-Helman, Department of Paediatrics and Allergy, Korczak Paediatric Centre, Copernicus

Memorial Hospital in Lodz, Medical University of Lodz, 71 Piłsudskiego St., 90-329 Lodz, Poland,

ORCID: 0000-0003-1787-6386, e-mail: asialechanska@poczta.onet.pl 

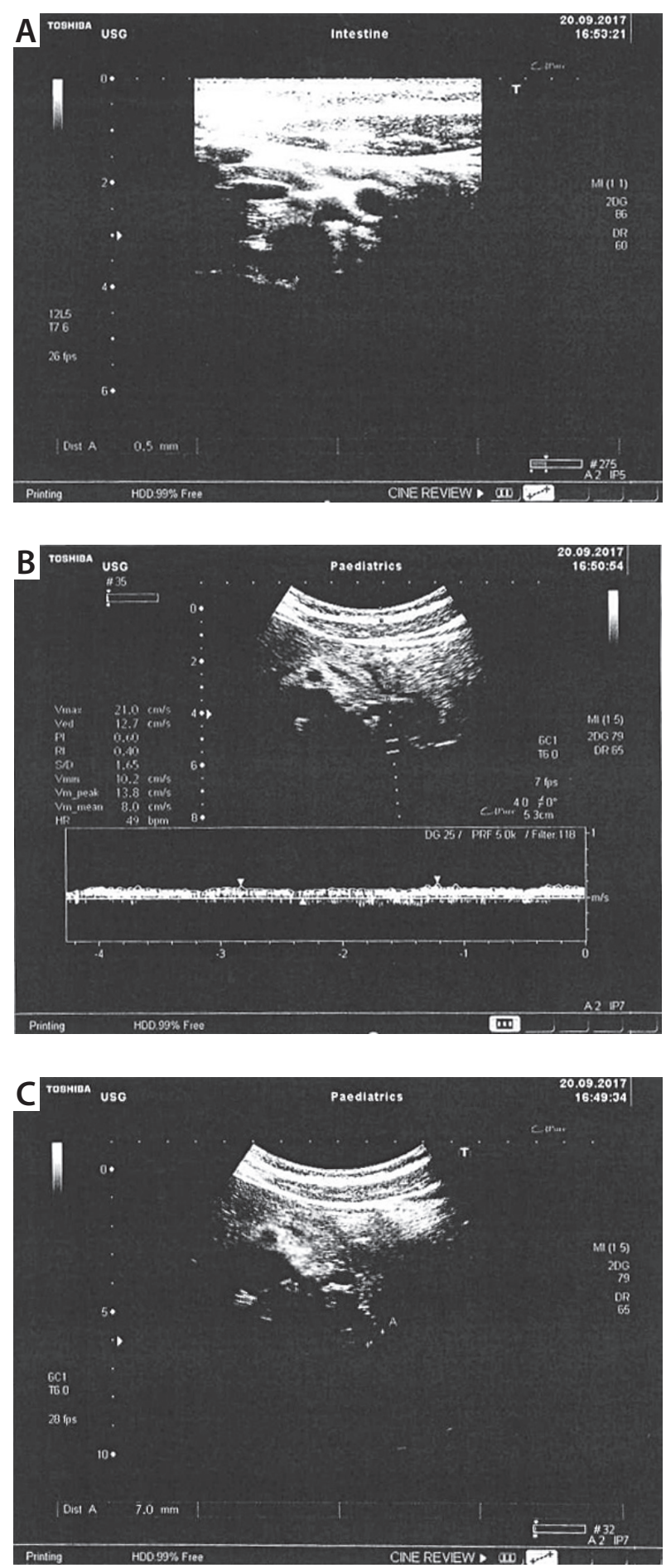

FIGURE 1. Doppler ultrasound revealing left renal vein dilatation up to the angle between the superior mesenteric artery and the aorta

erbated during eating and stress. No disorders were observed during the infancy period. There were no urinary diseases, kidneys stones, hearing loss, or gastrointestinal diseases in the patient's family medical history. Gastroscopy and ultrasonography were performed and revealed no abnormalities. Furthermore, bacterial, viral, and parasitic diseases were eliminated. Isolated erythrocyturia was observed in each laboratory test ordered by a general practitioner (GP) (10-15 erythrocytes/HPF).

\section{CLINICAL FINDINGS AND DIAGNOSTIC ASSESSMENT}

During admission to the hospital the general condition of the girl was good, and a physical examination revealed no deviations from the norm: blood pressure 100/65 mm Hg, body mass $49 \mathrm{~kg}-40 \mathrm{pc}$., growth $158 \mathrm{~cm}$ - 40 pc., and BMI $19.6 \mathrm{~kg} / \mathrm{m}^{2}-50 \mathrm{pc}$.

Renal ultrasonography (done twice) revealed that her kidneys were correctly positioned, and had the correct structure and size - both were $107 \mathrm{~mm}$ in the long axis. DUS revealed LRV dilatation up to the angle between the SMA and aorta with a diameter of $7 \mathrm{~mm}$ in the hilar region. This diameter was $0.5 \mathrm{~mm}$ between aorto-mesenteric vessels. Peak flow rate was $12 \mathrm{~cm} / \mathrm{s}$ in renal hilus, and flow between the aorta and spine was non-measurable due to aortic pulsation (Fig. 1). Findings from DUS were taken by a single paediatric radiologist.

To confirm our diagnosis, we conducted additional biochemical tests: blood urea nitrogen, creatinine $(0.6 \mathrm{mg} / \mathrm{dl})$, electrolytes, total protein, erythrocyte sedimentation rate, and C-reactive protein results were normal. During hospitalisation urinalysis was performed several times and revealed alternately occurring erythrocyturia and haematuria (20\% dysmorphic red blood cells). Daily calcium excretion was $1.57 \mathrm{mg} / \mathrm{kg} /$ day. Urine culture was negative.

Other tests: level of vitamin $\mathrm{D}$, anti-streptolysin $\mathrm{O}$, rheumatoid factor, immunoglobulin $\mathrm{A}, \mathrm{M}$, and $\mathrm{G}$, and complement component $(\mathrm{C} 3, \mathrm{C} 4)$ levels were normal. Antibody titres against: hepatitis $\mathrm{B}$ and $\mathrm{C}$ viruses, Yersinia, Toxocara canis, ascariasis, cytomegalovirus, Epstein-Barr virus, Borrelia burgdorferi, Toxoplasma gondii, and HIV were all negative. Prothrombin, partial thromboplastin, and bleeding times and complement levels were normal. Anti-nuclear factor and anti-double-stranded DNA antibodies were negative.

\section{TYPE OF INTERVENTION AND FOLLOW-UP}

According to the clinical picture, laboratory tests, and imaging studies, specialists decided that the 13-year-old girl should undergo medical surveillance.

The presented patient remains under nephrology clinical observation and takes no medication. No symptoms of NCS and adverse events have been observed.

\section{DISCUSSION}

The benefit of this case is the possibility to take a wide spectrum of laboratory tests and imaging studies. The official diagnostic criteria of NCS have not been established yet, and there are no simple procedures for a definitive diagnosis, which imposes a huge limitation on the whole diagnostic process. Therefore, diagnosis of haematuria can be prematurely finished or misdiagnosed. 
Left renal vein entrapment syndrome was reported first in 1950 by El-Sadr and Mina, but the term "nutcracker" is credited to de Schepper (1972), although it was used first by Chait et al. (1971) [2]. The most popular theory of NCS results from compression of the LRV between the aorta and the SMA, but different anatomical variants are possible. NCS can be diagnosed from childhood until the seventh decade of life, but it is more frequent in young adults, mostly in females [3]. Takebayashi et al. differentiate NCS into the following three subtypes: idiopathic renal bleeding, massive orthostatic proteinuria (with protein level $>400 \mathrm{mg} / \mathrm{dl}$ ), and severe orthostatic intolerance, which contributes to many difficulties in patients' everyday life [4]. Certain researchers point out that differential diagnosis of NCS should consider both proteinuria and haematuria [5].

NCS results in venous hypertension and congestion of the LRV with tributaries and left gonadal vein. Symptoms of NCS can be various, non-specific and include: haematuria, abdominal and left flank pain, pelvic congestion syndrome (lower abdominal pain, dysmenorrhoea, post-coital ache, dysuria, dyspareunia, pelvic, vulvar, gluteal or thigh varices), and orthostatic proteinuria. Sometimes haematuria may be the only disorder. Symptoms are usually aggravated by physical activity, stress, sitting, walking, or riding in a vehicle. In the presented case every urinalysis that has been conducted on the 13-year-old girl revealed alternately erythrocyturia and haematuria. She has been repeatedly examined by her GP and gastroenterologist, who did not see any abnormalities. Due to hospitalisation in specialist clinic, which was an opportunity to perform more advanced tests, the final diagnosis was established.

The primary diagnostic test should consist of the following: physical examination, collecting medical history (the most common symptoms, their evaluation, quality of life, perinatal history, pregnancy period, chronic diseases, current medication, family medical history), blood tests, urinalysis, urine culture, and abdominal ultrasonography. If these tests do not reveal any abnormalities, it is necessary to look further and exclude parasites, and viral and bacterial infections.

The diagnosis of NCS can be established by DUS. Peak velocity ratios between aorto-mesenteric and LRV hilar portion are usually used to diagnose NCS in DUS, and the cut-off value is 4.8 for the peak velocity ratio. DUS is a helpful method that can be used initially for detecting rare causes of haematuria [1]. Fitoz et al. reported that an upright position during DUS may have an effect on the LRV haemodynamics. What is more, researchers suggest new criteria in the diagnosis of NCS, adapted to the position of the body: in the supine position, 4.23 for the peak velocity ratio. Additional measurement of the SMA angle may be a useful tool in the diagnosis of NCS because SMA branching can affect development of NCS.
Furthermore, researchers suggest that NCS is more frequent in tall children, and BMI can also affect the SMA angle [6].

Additionally, CT angiography, venography, or magnetic resonance imaging can be helpful in making a diagnosis. In some cases, renal biopsy might be necessary to make a final diagnosis and to assess if there can be another cause of haematuria [7].

Treatment should be based on severity, stage of symptoms, and patient's age. In most cases haematuria decreases spontaneously (75\% of young patients) or completely disappears. For children diagnosed with NCS, a two-year observation time is recommended. Patients, especially in puberty, with mild symptoms may be followed conservatively [8]. In other cases, especially in patients with increasing pain, recurrent gross haematuria, and progressive renal function impairment, there are indications for surgical intervention, which may relieve the symptoms. An intravascular or extravascular stent placed in the compressed vein improves the blood flow. Sometimes intra-pelvic chemical cauterisation is proposed for the treatment, which has good effects and seldom causes complications [9].

The presented patient suffered from haematuria, and chronic back and abdominal pain, without any other abnormalities. It appears that NCS is underdiagnosed, and the path to the correct diagnosis can be long. Sixty-nine per cent of paediatric cases with isolated haematuria cannot be identified by routine tests.

\section{CONCLUSIONS}

NCS can be easily missed by routine tests conducted on children. The severity of clinical symptoms varies depending on the stage of the pathological process. Every patient with isolated haematuria should be diagnosed and treated by a specialist. In every case of long-lasting haematuria, to avoid delayed diagnosis, routine blood or urine tests should be conducted along with an image scan via DUS. The best treatment option, as in the case of the 13-year-old girl, is a conservative approach with regular medical observation without any medications.

\section{DISCLOSURE}

The authors declare no conflict of interest.

\section{REFERENCES}

1. Genc G, Ozkaya O. A Rare Cause of Recurrent Hematuria in Children: Nutcracker Syndrome. J Tropical Ped 2010; 56: 275-277.

2. El-Sadr AR, Mina E. Anatomical and surgical aspects in the operative management of varicocele. Urol Cutaneous Rev 1950; 54: 257-262.

3. Kurklinsky A, Rooke T. Nutcracker Phenomenon and Nutcracker Syndrome. Mayo Clin Proc 2010; 85: 552-559. 
4. Takebayashi S, Ueki T, Ikeda N, et al. Diagnosis of the nutcracker syndrome with color Doppler sonography. Am J Roentgenol 1999; 172: 39-43.

5. Ekim M, Ozcakar ZB, Fitoz S, et al. The "nutcracker phenomenon" with orthostatic proteinuria: case reports. Clin Nephrol 2006; 65: 280-283.

6. Fitoz S, Ekim M, Ozcakar ZB, et al. Nutcracker syndrome in children: the role of upright position examination and superior mesenteric artery angle measurement in the diagnosis. J Ultrasound Med 2007; 26: 573-580.

7. Ozono Y, Harada T, Namie S, et al. The "nutcracker" phenomenon in combination with IgA nephropathy. J Int Med Res 1995; 23: 126-131.

8. Siddiqui WJ, Bakar A, Aslam M, et al. Left Renal Vein Compression Syndrome: Cracking the Nut of Clinical Dilemmas - Three Cases and Review of Literature. Am J Case Rep 2017; 18: 754-759.

9. Chen Y, Wang I. Nutcracker Syndrome: An Overlooked Cause of Hematuria. Chang Gung Med 2002; 25: 700-705. 